\title{
Predictors of the development of nab-paclitaxel- induced peripheral neuropathy in breast cancer patients: post hoc analysis of a prospective, phase II, self-controlled clinical trial
}

Yuko Kanbayashi ( $\nabla$ yuko.kambayashi@ompu.ac.jp )

Osaka Medical and Pharmaceutical University: Osaka Ika Yakka Daigaku https://orcid.org/0000-00024680-5133

Koichi Sakaguchi

Kyoto Prefectural University of Medicine: Kyoto Furitsu Ika Daigaku

Takeshi Ishikawa

Kyoto Prefectural University of Medicine: Kyoto Furitsu Ika Daigaku

\section{Yusuke Tabuchi}

Kyoto Prefectural University of Medicine: Kyoto Furitsu Ika Daigaku

\section{Ryo Takagi}

Hokudai: Hokkaido Daigaku

\section{Isao Yokota}

Hokudai: Hokkaido Daigaku

\section{Norito Katoh}

Kyoto Prefectural University of Medicine: Kyoto Furitsu Ika Daigaku

\section{Koichi Takayama}

Kyoto Prefectural University of Medicine: Kyoto Furitsu Ika Daigaku

\section{Tetsuya Taguchi}

Kyoto Prefectural University of Medicine: Kyoto Furitsu Ika Daigaku

\section{Research Article}

Keywords: Nab-paclitaxel, Chemotherapy-induced peripheral neuropathy, Smoking, Breast cancer-affected side, BMI, Advanced age

Posted Date: April 19th, 2022

DOI: https://doi.org/10.21203/rs.3.rs-1263936/v2

License: (c) (1) This work is licensed under a Creative Commons Attribution 4.0 International License. Read Full License 
Page 2/19 


\section{Abstract}

In a previous study, we showed that cryotherapy and compression therapy have comparable efficacy in preventing nab-paclitaxel-induced peripheral neuropathy. However, even with cryotherapy or compression therapy, there were patients with National Cancer Institute Common Terminology Criteria for Adverse Events (CTCAE) version 4.0 grade $\geq 2$ and/or Patient Neurotoxicity Questionnaire (PNQ) grade $\geq D$ peripheral neuropathies. Therefore, this post hoc analysis was performed to identify predictors of nabpaclitaxel-induced peripheral neuropathy. The clinical data in this post hoc analysis were the data of 38 breast cancer patients receiving chemotherapy with nanoparticle albumin-bound paclitaxel (nab-PTX) at our outpatient chemotherapy center from August 2017 to March 2019. The number of patients was analyzed assuming that there were data for 76 hands. Variables related to the development of nab-PTXinduced peripheral neuropathy were used for regression analysis. Multivariate ordered logistic regression analysis was performed to identify predictors for the development of nab-PTX-induced peripheral neuropathy. Significant factors included smoking history [odds ratio $(O R)=4.64,95 \%$ confidence interval $(\mathrm{Cl})=1.60-13.5 ; P=0.0048]$ with neuropathy evaluated by CTCAE, body mass index (BMI) $(\mathrm{OR}=1.13$, $95 \% \mathrm{Cl}=1.01-1.26 ; P=0.039)$ with neuropathy evaluated by PNQ (sensory), and smoking history (OR = $3.80,95 \% \mathrm{Cl}=1.40-10.30 ; P=0.0087)$ and age $(\mathrm{OR}=1.06,95 \% \mathrm{Cl}=1.01-1.11 ; P=0.012)$ with neuropathy evaluated by PNQ (motor). In conclusion, smoking history, BMI and age were identified as significant predictors of the development of nab-PTX-induced-peripheral neuropathy.

\section{Introduction}

Chemotherapy-induced peripheral neuropathy (CIPN) can become a major drug-induced adverse reaction that becomes a dose-limiting toxicity of chemotherapy [1-5]. In recent years, the efficacy of cryotherapy using frozen gloves (FGs) and compression therapy using surgical gloves (SGs) to prevent taxaneinduced peripheral neuropathy has been reported [6-8]. However, no reports appear to have compared the efficacy of cryotherapy and compression therapy. We therefore planned a prospective, self-controlled trial to compare the efficacy of cryotherapy using FGs and compression therapy using SGs to prevent nanoparticle albumin-bound paclitaxel (nab-PTX) -induced peripheral neuropathy [9]. It was found that cryotherapy and compression therapy showed comparable efficacy in preventing CIPN. However, even with cryotherapy or compression therapy, there were patients with National Cancer Institute Common Terminology Criteria for Adverse Events (CTCAE) version 4.0 grade $\geq 2(18.4 \%)$ and/or Patient Neurotoxicity Questionnaire (PNQ) grade $\geq$ D peripheral neuropathies [9]. As for taxane-based chemotherapy, previous study reported advanced age, diabetes, alcohol, obesity and menopause are predictors for CIPN[10-12]. Duloxetine, which is currently recommended for the treatment of painful neuropathy in the guidelines $[13,14]$. However, Smith et al. state that duloxetine is more effective in CIPN with platinum-based anticancer drugs than with taxanes [15]. Various studies have statistically analyzed the predictive factors for CIPN [10-12]. Nevertheless, effective strategies for managing CIPN among affected patients remain elusive. Thus, in addition to improving the quality of life for patients undergoing 
chemotherapy, there is an unmet need to better-identify patients at risk for CIPN. Therefore, a sub-analysis to identify predictors of nab-PTX-induced peripheral neuropathy was performed.

\section{Patients And Methods}

\section{Study design and participants}

This is a post-hoc analysis of a previously published a prospective, phase II, self-controlled clinical trial conducted between August 2017 and March 2019 (registered with the University Hospital Medical Information Network, date of first registration 24/12/2017: UMIN000030536). (https://upload.umin.ac.jp/cgi-open-bin/ctr/ctr_view.cgi?recptno=R000034856) [9]. This trial was approved by the Medical Ethics Review Committee of Kyoto Prefectural University of Medicine, in accordance with the tenets of the Declaration of Helsinki (approval no. ERB-C-906-1). Written, informed consent was obtained from all patients prior to their enrollment including possibility to use data for post hoc analysis. Exclusion criteria were as follows: peripheral sensory/motor neuropathy (CTCAE grade $\geq 2$ ), underlying diseases that could potentially cause peripheral neuropathy (such as diabetes mellitus or postherpetic neuralgia), allergy to the material of FGs (polyurethane and nylon) or the material of SGs (latex), brain metastases, or any other reasons based on the judgment of the primary physician. Breast cancer patients who received $260 \mathrm{mg} / \mathrm{m}^{2}$ of nab-PTX as 30-min intravenous infusion every 3 weeks for four cycles were eligible to participate in this trial. Human epidermal growth factor receptor 2 (HER2)positive patients received trastuzumab after administration of nab-PTX during each cycle. Patients wore an FG on one hand and two SGs of the same size (i.e., one size smaller than the size that best fit their hand) on the other hand during chemotherapy.

\section{Evaluation of Peripheral Neuropathy}

Peripheral neuropathy was evaluated by treating pharmacists and/or nurses using NCl-CTCAE version 4.0 at the following times: pretreatment (baseline); just before each treatment cycle; and six weeks after chemotherapy completion ( \pm 2 weeks). Peripheral neuropathy was also evaluated using the Japanese version of the PNQ, a validated patient-reported questionnaire on neuropathy and activities of daily living that correlates with quality of life [16]. The PNQ comprises two items (sensory and motor neuropathy). The subjective responses to each item are graded from $A$ to $E$ by the patient. Each item of the PNQ was defined as A (no neuropathy), B (mild neuropathy), C (moderate neuropathy that does not interfere with activities of daily living ( $A D L), D$ (moderate neuropathy that interferes with $A D L$ ), or $E$ (severe neuropathy that interferes with $A D L)$. In this trial, $P N Q$ grades were coded as $1-5$, with higher scores indicating more severe peripheral neuropathy. In this post hoc analysis, the values of CTCAE (sensory and motor grades) and PNQ (sensory and motor grades) at the final evaluation were used as the objective variables. As for CTCAE, the same result was obtained for sensory and motor grades, so in the present study, the objective variables that were analyzed were CTCAE, PNQ (sensory), and PNQ (motor).

\section{Evaluation of Fingertip Temperatures}


To investigate mechanisms underlying protection against peripheral neuropathy, the temperature at each fingertip was measured by thermography at the first cycle of nab-PTX. Temperatures at each fingertip on both hands were measured before wearing gloves and $30 \mathrm{~min}$ after the end of nab-PTX infusion using a thermographic camera (INFRA-EYE 2000; Fujitsu, Tokyo, Japan).

\section{Explanatory variables}

The following patient data were collected and analyzed to identify the predictors for CIPN. Variables evaluated included factors that could potentially impact the development of CIPN: demographic data [age, height, weight, and body mass index (BMI)], Eastern Cooperative Oncology Group performance status, smoking history, presence of comorbidity (diabetes mellitus), the dominant side hand on the breast cancer-affected side, combination therapy with trastuzumab, surgical glove size, average fingertip temperature after administration, and average difference in fingertip temperature before and after administration. The clinical information before the first dose of nab-PTX was used.

\section{Statistical Analysis}

The analysis was performed assuming that each hand was independent. Since there is a possibility of a correlation between two hands of the same individual, a univariate analysis was performed by adding the patient identification (ID) number to the explanatory variable.

Explanatory variables were examined for multicollinearity (correlation coefficient $|r| \geq 0.7$ ), since when correlations exist among the variables, this can lead to incorrect results of regression analyses.

Explanatory variables were selected based on correlation strength with the level of the CIPN (objective variable) or clinical significance. First, univariate ordered logistic regression analysis between the outcomes and each potential explanatory variable was performed. Subsequently, a multivariate ordered logistic regression model was constructed using the stepwise backward selection procedure with the potential candidate variables. Ordered logistic regression analysis was performed, because the level of CIPN was evaluated by a graded scale, and multiple factors potentially involved as predictors for the development of CIPN had to be evaluated simultaneously.

For all statistical analyses, values of $P<0.05$ (2-tailed) were considered significant. All analyses were performed using JMP version 14.3.0. (SAS Institute, Cary, NC, USA).

\section{Results}

Between August 2017 and March 2019, a total of 43 patients with breast cancer were enrolled, of whom 38 were evaluated in this trial [9]. Table 1-1, 1-2, and 1-3 presents the clinical characteristics for a total of 76 hands (38 patients), the potential variables related to the development of CIPN, and the results of univariate analysis evaluating CTCAE, PNQ (sensory) and PNQ (motor), respectively. Although surgical glove size and average difference in fingertip temperature before and after administration were significant predictors on univariate analysis, they were not used for multivariate analysis due to missing data. Multicollinearity was observed in body weight and BMI. BMI, which was considered the most 
clinically relevant predictor for CIPN, was used in the analysis. The backward stepwise selection procedure identified two variables (smoking history, the dominant side hand on the breast cancer-affected side) with neuropathy evaluated by CTCAE, two variables (smoking history and $\mathrm{BMI}$ ) with neuropathy evaluated by PNQ (sensory) and two variables (smoking history and age) with neuropathy evaluated by PNQ (motor). 
Table 1

1. Clinical characteristics, extracted variables, and results of univariate analyses for CTCAE $(n=76)$

$\begin{array}{llllll}\text { Grade } & \text { Grade } & \text { Grade } & \text { Grade } & \begin{array}{l}P \\ \text { value }\end{array} & \begin{array}{l}\text { Odds } \\ \text { ratio }\end{array} \\ (n= & 1 & 2 & 3 & & (n= \\ 16) & 46) & (n= & (n=4) & & (95 \%) \\ & & & & \text { Cl })\end{array}$

Demographic data

Age (y), median (range)

59

58

61

66

0.29

1.02

(45-

(34-

(40-

(62-

73)

76)

66)

70)

(0.98-

157

156

159

157

0.37

1.07)

Height (cm), median (range)

(154-

(148-

(155-

(151-

159)

165)

162)

163)

1.05

Weight $(\mathrm{kg})$, median (range)

47.8

54

64.6

44.4

1.15)

$\operatorname{BMI}\left(\mathrm{kg} / \mathrm{m}^{2}\right)$, median (range)

$\begin{array}{lllll}(41.7- & (41.7- & (47- & (36.8- & (0.99- \\ 67.5) & 79.3) & 67) & 52) & 1.09)\end{array}$

PS $(0 / 1)$

$\begin{array}{llll}19.5 & 22.2 & 25.5 & 17.9 \\ (17.2- & (15.7- & (17.9- & (16.1- \\ 27.3) & 30.6) & 27.7) & 19.7)\end{array}$

$0.27 \quad 1.07$

$16 / 0$

$44 / 2$

$10 / 0$

$2 / 2$

$(0.95-$
$1.20)$

\begin{tabular}{|lllllll|} 
& & & & & & (1.59- \\
Smoking history, $\mathrm{n}(\%)$ & 2 & 12 & 4 & 4 & $0.004^{*}$ & 4.79 \\
& $(12.5)$ & $(26.1)$ & $(40.0)$ & $(100.0)$ & $\begin{array}{c}(1.65- \\
13.92)\end{array}$ \\
\hline $\begin{array}{l}\text { The dominant side hand on the breast } \\
\text { cancer-affected side, } \mathrm{n}(\%)\end{array}$ & 6 & 26 & 8 & 2 & 0.085 & 2.27 \\
& $(37.5)$ & $(56.5)$ & $(80.0)$ & $(50.0)$ & & $(0.89-$ \\
$\begin{array}{l}\text { Combination therapy with trastuzumab, } \\
\mathrm{n}(\%)\end{array}$ & 6 & 20 & 4 & 2 & 0.62 & 1.26 \\
& $(37.5)$ & $(43.5)$ & $(40.0)$ & $(50.0)$ & & $(0.51-$ \\
\hline Treatment & & & & & & $3.11)$ \\
\hline
\end{tabular}

CTCAE, the National Cancer Institute Common Terminology Criteria for Adverse Events; $\mathrm{Cl}$, confidence interval; BMI, body mass index; PS, Performance Status; SD, standard deviation

$\star P<0.05$ 


\begin{tabular}{|c|c|c|c|c|c|c|}
\hline & $\begin{array}{l}\text { Grade } \\
0 \\
(n= \\
16)\end{array}$ & $\begin{array}{l}\text { Grade } \\
1 \\
(n= \\
46)\end{array}$ & $\begin{array}{l}\text { Grade } \\
2 \\
(n= \\
10)\end{array}$ & $\begin{array}{l}\text { Grade } \\
3 \\
(n=4)\end{array}$ & $\begin{array}{l}P \\
\text { value }\end{array}$ & $\begin{array}{l}\text { Odds } \\
\text { ratio } \\
(95 \% \\
\text { Cl) }\end{array}$ \\
\hline Neo-adjuvant, n (\%) & $\begin{array}{l}10 \\
(62.5)\end{array}$ & $\begin{array}{l}30 \\
(65.2)\end{array}$ & $\begin{array}{l}6 \\
(60.0)\end{array}$ & $\begin{array}{l}4 \\
(100.0)\end{array}$ & 0.51 & $\begin{array}{l}1.38 \\
(0.54- \\
3.54)\end{array}$ \\
\hline Adjuvant, n (\%) & $\begin{array}{l}8 \\
(50.0)\end{array}$ & $\begin{array}{l}10 \\
(21.7)\end{array}$ & $\begin{array}{l}4 \\
(40.0)\end{array}$ & 0 & 0.11 & $\begin{array}{l}0.44 \\
(0.16- \\
1.20)\end{array}$ \\
\hline Palliative, n (\%) & 0 & $4(8.7)$ & 0 & 0 & 0.87 & $\begin{array}{l}1.19 \\
(0.16- \\
8.78)\end{array}$ \\
\hline Surgical glove size, mean \pm SD & $\begin{array}{l}5.69 \pm \\
0.25\end{array}$ & $\begin{array}{l}5.85 \pm \\
0.38\end{array}$ & $\begin{array}{l}6.00 \pm \\
0.33\end{array}$ & $\begin{array}{l}5.75 \pm \\
0.29\end{array}$ & $0.017 *$ & $\begin{array}{l}5.67 \\
(1.37- \\
23.50)\end{array}$ \\
\hline $\begin{array}{l}\text { Average fingertip temperature after } \\
\text { administration }\left({ }^{\circ} \mathrm{C}\right) \text {, median (range) }\end{array}$ & $\begin{array}{l}23.9 \\
(15.3- \\
30.6)\end{array}$ & $\begin{array}{l}28.5 \\
(12.6- \\
32.6)\end{array}$ & $\begin{array}{l}29.0 \\
(17- \\
31.1)\end{array}$ & $\begin{array}{l}28.5 \\
(27.6- \\
29.5)\end{array}$ & 0.095 & $\begin{array}{l}1.10 \\
(0.98- \\
1.24)\end{array}$ \\
\hline $\begin{array}{l}\text { Average difference in fingertip } \\
\text { temperature } \\
\text { before and after administration }\left({ }^{\circ} \mathrm{C}\right) \text {, } \\
\text { median (range) }\end{array}$ & $\begin{array}{l}8.1 \\
(1.7- \\
16.3)\end{array}$ & $\begin{array}{l}3.3 \\
(0.2- \\
18.4)\end{array}$ & $\begin{array}{l}5.3 \\
(2.1- \\
11.8)\end{array}$ & $\begin{array}{l}5.1 \\
(4.1- \\
6.0)\end{array}$ & 0.14 & $\begin{array}{l}0.91 \\
(0.79- \\
1.03)\end{array}$ \\
\hline \multicolumn{7}{|c|}{$\begin{array}{l}\text { CTCAE, the National Cancer Institute Common Terminology Criteria for Adverse Events; Cl, confidence } \\
\text { interval; BMI, body mass index; PS, Performance Status; SD, standard deviation }\end{array}$} \\
\hline$\star P<0.05$ & & & & & & \\
\hline
\end{tabular}


Table 1

2. Clinical characteristics, extracted variables, and results of univariate analyses for PNQ (sensory) $(n=$ 76)

\begin{tabular}{|c|c|c|c|c|c|}
\hline & $\begin{array}{l}A \\
(n=16)\end{array}$ & $\begin{array}{l}B \\
(n= \\
46)\end{array}$ & $\begin{array}{l}\text { C, D or } \\
E \\
(n=10)\end{array}$ & $P$ value & $\begin{array}{l}\text { Odds } \\
\text { ratio } \\
(95 \% \mathrm{Cl})\end{array}$ \\
\hline \multicolumn{6}{|l|}{ Demographic data } \\
\hline \multirow[t]{2}{*}{ Age $(y)$, median (range) } & 55 & 60 & 63.5 & 0.084 & 1.04 \\
\hline & $(34-73)$ & $\begin{array}{l}(34- \\
76)\end{array}$ & $\begin{array}{l}(40- \\
70)\end{array}$ & & $\begin{array}{l}(0.99- \\
1.08)\end{array}$ \\
\hline \multirow[t]{2}{*}{ Height (cm), median (range) } & 157.3 & 155.9 & 162 & $0.041^{\star}$ & 1.12 \\
\hline & $\begin{array}{l}(151- \\
165)\end{array}$ & $\begin{array}{l}(148- \\
165)\end{array}$ & $\begin{array}{l}(151.1- \\
165)\end{array}$ & & $\begin{array}{l}(1.01- \\
1.22)\end{array}$ \\
\hline \multirow[t]{2}{*}{ Weight (kg), median (range) } & 50 & 50 & 62.5 & $0.0038 *$ & 1.07 \\
\hline & $\begin{array}{l}(41.7- \\
67.5)\end{array}$ & $\begin{array}{l}(41.7- \\
67)\end{array}$ & $\begin{array}{l}(36.8- \\
79.3)\end{array}$ & & $\begin{array}{l}(1.02- \\
1.13)\end{array}$ \\
\hline \multirow[t]{2}{*}{ BMI $\left(\mathrm{kg} / \mathrm{m}^{2}\right)$, median (range) } & 19.8 & 20.1 & 23.6 & $0.047^{*}$ & 1.12 \\
\hline & $27.3)^{-1}$ & $\begin{array}{l}(15.7- \\
30.6)\end{array}$ & $\begin{array}{l}(16.1- \\
29.1)\end{array}$ & & $\begin{array}{l}(1.01- \\
1.26)\end{array}$ \\
\hline \multirow[t]{2}{*}{ PS (0/1) } & $16 / 1$ & $38 / 1$ & $18 / 2$ & 0.45 & 2.09 \\
\hline & & & & & $\begin{array}{l}(0.31- \\
14.26)\end{array}$ \\
\hline \multirow[t]{2}{*}{ Smoking history, n (\%) } & 5 & 8 & 9 & 0.22 & 1.82 \\
\hline & $(29.4)$ & $(20.5)$ & $(45.0)$ & & $\begin{array}{l}(0.70- \\
4.71)\end{array}$ \\
\hline \multirow{2}{*}{$\begin{array}{l}\text { The dominant side hand on the breast } \\
\text { cancer-affected side, } \mathrm{n}(\%)\end{array}$} & 7 & 21 & 14 & 0.077 & 2.22 \\
\hline & $(41.2)$ & $(53.8)$ & (70) & & $\begin{array}{l}(0.92- \\
5.39)\end{array}$ \\
\hline \multirow{2}{*}{$\begin{array}{l}\text { Combination therapy with trastuzumab, } \mathrm{n} \\
(\%)\end{array}$} & 7 & 16 & 9 & 0.62 & 1.26 \\
\hline & $(41.2)$ & $(41.0)$ & $(45)$ & & $\begin{array}{l}(0.51- \\
3.11)\end{array}$ \\
\hline
\end{tabular}

PNQ, the Patient Neurotoxicity Questionnaire; Cl, confidence interval; BMI, body mass index; PS, Performance Status; SD, standard deviation 


\begin{tabular}{|c|c|c|c|c|c|}
\hline & $\begin{array}{l}\text { A } \\
(n=16)\end{array}$ & $\begin{array}{l}B \\
(n= \\
46)\end{array}$ & $\begin{array}{l}\text { C, D or } \\
E \\
(n=10)\end{array}$ & $P$ value & $\begin{array}{l}\text { Odds } \\
\text { ratio } \\
(95 \% \mathrm{Cl})\end{array}$ \\
\hline Neo-adjuvant, n (\%) & $\begin{array}{l}11 \\
(64.7)\end{array}$ & $\begin{array}{l}23 \\
(59.0)\end{array}$ & $\begin{array}{l}16 \\
(80.0)\end{array}$ & 0.30 & $\begin{array}{l}1.62 \\
(0.65- \\
4.01)\end{array}$ \\
\hline Adjuvant, n (\%) & $\begin{array}{l}6 \\
(35.3)\end{array}$ & $\begin{array}{l}13 \\
(33.3)\end{array}$ & $\begin{array}{l}3 \\
(15.0)\end{array}$ & 0.11 & $\begin{array}{l}0.44 \\
(0.16- \\
1.20)\end{array}$ \\
\hline Palliative, n (\%) & $\begin{array}{l}1 \\
(5.9)\end{array}$ & $\begin{array}{l}2 \\
(5.1)\end{array}$ & $\begin{array}{l}1 \\
(5.0)\end{array}$ & 0.87 & $\begin{array}{l}1.19 \\
(0.16- \\
8.78)\end{array}$ \\
\hline Surgical glove size, mean \pm SD & $\begin{array}{l}5.71 \pm \\
0.31\end{array}$ & $\begin{array}{l}5.82 \pm \\
0.39\end{array}$ & $\begin{array}{l}5.95 \pm \\
0.28\end{array}$ & $0.025^{\star}$ & $\begin{array}{l}4.77 \\
(1.22- \\
18.70)\end{array}$ \\
\hline $\begin{array}{l}\text { Average fingertip temperature } \\
\text { after administration }\left({ }^{\circ} \mathrm{C}\right) \text {, median (range) }\end{array}$ & $\begin{array}{l}21.0 \\
(15.3- \\
26.9)\end{array}$ & $\begin{array}{l}29.0 \\
(12.6- \\
32.6)\end{array}$ & $\begin{array}{l}31.1 \\
(13.3- \\
31.1)\end{array}$ & 0.082 & $\begin{array}{l}1.12 \\
(0.99- \\
1.24)\end{array}$ \\
\hline $\begin{array}{l}\text { Average difference in fingertip temperature } \\
\text { before and after administration }\left({ }^{\circ} \mathrm{C}\right) \text {, } \\
\text { median (range) }\end{array}$ & $\begin{array}{l}11.5 \\
(3.3-16.3)\end{array}$ & $\begin{array}{l}3.5 \\
(0.9- \\
18.4)\end{array}$ & $\begin{array}{l}4.1 \\
(0.2- \\
11.8)\end{array}$ & $0.018^{*}$ & $\begin{array}{l}0.84 \\
(0.73- \\
0.97)\end{array}$ \\
\hline \multicolumn{6}{|c|}{$\begin{array}{l}\text { PNQ, the Patient Neurotoxicity Questionnaire; Cl, confidence interval; BMI, body mass index; PS, } \\
\text { Performance Status; SD, standard deviation }\end{array}$} \\
\hline$\star P<0.05$ & & & & & \\
\hline
\end{tabular}


Table 1

3. Clinical characteristics, extracted variables, and results of univariate analyses for PNQ (motor) $(n=76)$

\begin{tabular}{|c|c|c|c|c|c|}
\hline & $\begin{array}{l}A \\
(n=41)\end{array}$ & $\begin{array}{l}B \\
(n= \\
26)\end{array}$ & $\begin{array}{l}C, D \text { or } E \\
(n=9)\end{array}$ & $P$ value & 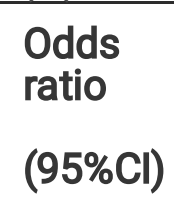 \\
\hline \multicolumn{6}{|l|}{ Demographic data } \\
\hline Age (y), median (range) & $\begin{array}{l}58 \\
(34-69)\end{array}$ & $\begin{array}{l}61(40- \\
76)\end{array}$ & $\begin{array}{l}65(61- \\
70)\end{array}$ & $0.013^{*}$ & $\begin{array}{l}1.06 \\
(1.01- \\
1.11)\end{array}$ \\
\hline Height (cm), median (range) & $\begin{array}{l}157.3 \\
(148- \\
165)\end{array}$ & $\begin{array}{l}156.8 \\
(148- \\
165)\end{array}$ & $\begin{array}{l}158.3 \\
(151.1- \\
162.5)\end{array}$ & 0.96 & $\begin{array}{l}1.00 \\
(0.91- \\
1.10)\end{array}$ \\
\hline Weight (kg), median (range) & $\begin{array}{l}54 \\
(41.7- \\
67.5)\end{array}$ & $\begin{array}{l}50.5 \\
(41.7- \\
79.3)\end{array}$ & $\begin{array}{l}64.6 \\
(36.8- \\
67)\end{array}$ & 0.48 & $\begin{array}{l}1.02 \\
(0.97- \\
1.06)\end{array}$ \\
\hline $\mathrm{BMI}\left(\mathrm{kg} / \mathrm{m}^{2}\right)$, median (range) & $\begin{array}{l}20.1 \\
(15.7- \\
30.6)\end{array}$ & $\begin{array}{l}21.4 \\
(17.2- \\
29.1)\end{array}$ & $\begin{array}{l}24.8 \\
(16.1- \\
27.2)\end{array}$ & 0.62 & $\begin{array}{l}1.03 \\
(0.92- \\
1.15)\end{array}$ \\
\hline PS $(0 / 1)$ & $39 / 2$ & $26 / 0$ & $7 / 2$ & 0.24 & $\begin{array}{l}3.10 \\
(0.47- \\
20.47)\end{array}$ \\
\hline Smoking history, n (\%) & $\begin{array}{l}7 \\
(17.1)\end{array}$ & $\begin{array}{l}10 \\
(38.5)\end{array}$ & $\begin{array}{l}5 \\
(55.6)\end{array}$ & $0.0093^{\star}$ & $\begin{array}{l}3.65 \\
(1.38- \\
9.69)\end{array}$ \\
\hline $\begin{array}{l}\text { The dominant side hand on the breast } \\
\text { cancer-affected side, } \mathrm{n}(\%)\end{array}$ & $\begin{array}{l}21 \\
(51.2)\end{array}$ & $\begin{array}{l}17 \\
(65.4)\end{array}$ & $\begin{array}{l}4 \\
(44.4)\end{array}$ & 0.65 & $\begin{array}{l}1.22 \\
(0.51- \\
2.96)\end{array}$ \\
\hline $\begin{array}{l}\text { Combination therapy with trastuzumab, } n \\
(\%)\end{array}$ & $\begin{array}{l}17 \\
(41.5)\end{array}$ & $\begin{array}{l}11 \\
(42.3)\end{array}$ & $\begin{array}{l}4 \\
(44.4)\end{array}$ & 0.86 & $\begin{array}{l}1.08 \\
(0.45- \\
2.61)\end{array}$ \\
\hline Treatment & & & & & \\
\hline
\end{tabular}

PNQ, the Patient Neurotoxicity Questionnaire; $\mathrm{Cl}$, confidence interval; BMI, body mass index; PS, Performance Status; SD, standard deviation

$\star P<0.05$ 


\begin{tabular}{|c|c|c|c|c|c|}
\hline & $\begin{array}{l}\text { A } \\
(n=41)\end{array}$ & $\begin{array}{l}B \\
(n= \\
26)\end{array}$ & $\begin{array}{l}C, D \text { or } E \\
(n=9)\end{array}$ & $P$ value & $\begin{array}{l}\text { Odds } \\
\text { ratio } \\
(95 \% \mathrm{Cl})\end{array}$ \\
\hline Neo-adjuvant, n (\%) & $\begin{array}{l}28 \\
(68.3)\end{array}$ & $\begin{array}{l}14 \\
(53.8)\end{array}$ & $\begin{array}{l}8 \\
(88.9)\end{array}$ & 0.98 & $\begin{array}{l}1.01 \\
(0.41- \\
2.54)\end{array}$ \\
\hline Adjuvant, n (\%) & $\begin{array}{l}10 \\
(24.4)\end{array}$ & $\begin{array}{l}11 \\
(42.3)\end{array}$ & $\begin{array}{l}1 \\
(11.1)\end{array}$ & 0.63 & $\begin{array}{l}1.27 \\
(0.49- \\
3.30)\end{array}$ \\
\hline Palliative, n (\%) & $\begin{array}{l}3 \\
(7.3)\end{array}$ & $\begin{array}{l}1 \\
(3.8)\end{array}$ & 0 & 0.38 & $\begin{array}{l}0.35 \\
(0.034- \\
3.61)\end{array}$ \\
\hline Surgical glove size, mean \pm SD & $\begin{array}{l}5.82 \pm \\
0.40\end{array}$ & $\begin{array}{l}5.81 \pm \\
0.29\end{array}$ & $\begin{array}{l}5.94 \pm \\
0.30\end{array}$ & 0.47 & $\begin{array}{l}1.63 \\
(0.43- \\
6.17)\end{array}$ \\
\hline $\begin{array}{l}\text { Average fingertip temperature } \\
\text { after administration }\left({ }^{\circ} \mathrm{C}\right) \text {, median (range) }\end{array}$ & $\begin{array}{l}26.1 \\
(12.6- \\
32.6)\end{array}$ & $\begin{array}{l}29.1 \\
(17.0- \\
31.5)\end{array}$ & $\begin{array}{l}29.5 \\
(13.3- \\
29.5)\end{array}$ & 1.00 & $\begin{array}{l}1.00 \\
(0.89- \\
1.12)\end{array}$ \\
\hline $\begin{array}{l}\text { Average difference in fingertip } \\
\text { temperature } \\
\text { before and after administration }\left({ }^{\circ} \mathrm{C}\right) \text {, } \\
\text { median (range) }\end{array}$ & $\begin{array}{l}5.2 \\
(0.2- \\
18.4)\end{array}$ & $\begin{array}{l}4.7 \\
(1.5- \\
13.5)\end{array}$ & $\begin{array}{l}5.8 \\
(0.6- \\
9.3)\end{array}$ & 0.55 & $\begin{array}{l}0.96 \\
(0.83- \\
1.10)\end{array}$ \\
\hline \multicolumn{6}{|c|}{$\begin{array}{l}\text { PNQ, the Patient Neurotoxicity Questionnaire; Cl, confidence interval; BMI, body mass index; PS, } \\
\text { Performance Status; SD, standard deviation }\end{array}$} \\
\hline$\star P<0.05$ & & & & & \\
\hline
\end{tabular}


Table 2

- 1 Results of multivariate ordered logistic regression analysis for CTCAE ( $n=76$, accuracy 46/76)

\begin{tabular}{|c|c|c|c|c|}
\hline \multirow[t]{2}{*}{ Variable } & \multirow[t]{2}{*}{$P$ value } & \multirow{2}{*}{$\begin{array}{l}\text { Odds } \\
\text { ratio }\end{array}$} & \multicolumn{2}{|l|}{$95 \% \mathrm{Cl}$} \\
\hline & & & $\begin{array}{l}\text { Lower } \\
95 \%\end{array}$ & $\begin{array}{l}\text { Upper } \\
95 \%\end{array}$ \\
\hline Smoking history & $0.0048 *$ & 4.64 & 1.60 & 13.5 \\
\hline $\begin{array}{l}\text { The dominant side hand on the breast cancer- } \\
\text { affected side }\end{array}$ & 0.081 & 2.29 & 0.90 & 5.79 \\
\hline \multicolumn{5}{|l|}{$\mathrm{Cl}$, confidence interval } \\
\hline$\star P<0.05$ & & & & \\
\hline
\end{tabular}

Table 2

2 Results of multivariate ordered logistic regression analysis for PNQ (sensory) ( $n=76$, accuracy $43 / 76$ )

\begin{tabular}{|c|c|c|c|c|}
\hline \multirow[t]{2}{*}{ Variable } & \multirow[t]{2}{*}{$P$ value } & \multirow[t]{2}{*}{ Odds ratio } & \multicolumn{2}{|l|}{$95 \% \mathrm{Cl}$} \\
\hline & & & Lower 95\% & Upper $95 \%$ \\
\hline Smoking history & 0.16 & 1.99 & 0.76 & 5.23 \\
\hline BMI & $0.039 *$ & 1.13 & 1.01 & 1.26 \\
\hline \multicolumn{5}{|c|}{$\mathrm{Cl}$, confidence interval; $\mathrm{BMI}$, body mass index } \\
\hline$\star P<0.05$ & & & & \\
\hline
\end{tabular}

Table 2

3 Results of multivariate ordered logistic regression analysis for PNQ (motor) ( $n=76$, accuracy 48/76)

\begin{tabular}{|c|c|c|c|c|}
\hline \multirow[t]{2}{*}{ Variable } & \multirow[t]{2}{*}{$P$ value } & \multirow[t]{2}{*}{ Odds ratio } & \multicolumn{2}{|l|}{$95 \% \mathrm{Cl}$} \\
\hline & & & Lower $95 \%$ & Upper $95 \%$ \\
\hline Smoking history & $0.0087 *$ & 3.80 & 1.40 & 10.30 \\
\hline Age & $0.012^{\star}$ & 1.06 & 1.01 & 1.11 \\
\hline \multicolumn{5}{|c|}{$\mathrm{Cl}$, confidence interval; SBP, Systolic Blood Pressure; RAS, renin-angiotensin system } \\
\hline \multicolumn{5}{|l|}{$\star P<0.05$} \\
\hline
\end{tabular}


These variables were then entered into the multivariate ordered logistic regression analysis. Significant factors identified for the development of CIPN included smoking history [odds ratio (OR) $=4.64,95 \%$ confidence interval $(\mathrm{Cl})=1.60-13.5 ; P=0.0048]$ with neuropathy evaluated by CTCAE (Table $2-1)$, BMI $(\mathrm{OR}=1.13,95 \% \mathrm{Cl}=1.01-1.26 ; P=0.039)$ with neuropathy evaluated by PNQ (sensory) (Table <link rid="tb6">2</link>-2), and smoking history $(\mathrm{OR}=3.80,95 \% \mathrm{Cl}=1.40-10.30 ; P=0.0087)$ and age $(\mathrm{OR}=$ $1.06,95 \% \mathrm{Cl}=1.01-1.11 ; P=0.012$ ) with neuropathy evaluated by PNQ (motor) (Table 2-3). The accuracy of our model was 46/76 (evaluated by CTCAE), 43/76 [evaluated by PNQ (sensory)] and 48/76 [evaluated by PNQ (motor)], respectively. The accuracy was determined as the ratio of the patients whose expected value was equal to the observed value (Table 2 ).

\section{Discussion}

The multivariate ordered logistic regression analysis performed in the present study showed that the significant predictors for the development of nab-PTX-induced peripheral neuropathy included smoking history with neuropathy evaluated by CTCAE, BMI with neuropathy evaluated by PNQ (sensory), and smoking history and advanced age with neuropathy evaluated by PNQ (motor). The dominant side hand on the breast cancer-affected side tended to develop CIPN evaluated by CTCAE. Surgical glove size and average difference in fingertip temperature before and after administration were also predictors on univariate analysis.

Several studies have reported that smoking was the predictor for CIPN development [17-20]. The result of the current study is consistent with this previous finding. Thus, clinicians need to know that the incidence and severity of CIPN are higher in patients with a smoking history.

Greenlee et al. in their prospective, observational, cohort study showed that obesity was associated with CIPN in breast cancer patients who received taxane treatment [21]. In this study, BMI was a predictor for CIPN development. The result of the current study is consistent with this previous finding [21-24]. Clinicians need to pay particular attention to the development of CIPN in obese patients.

Furthermore, the present study also found that advanced age is a predictor for CIPN $[19,25]$. This result is consistent with previous studies. Therefore, clinicians also should be aware that the risk of CIPN increases with age.

In addition, the current study, dominant side hand on the breast cancer-affected side tended to develop CIPN evaluated by CTCAE, though it was not significant. This result was also consistent with findings in routine clinical practice. Therefore, clinicians should be aware that, in breast cancer patients, the risk of developing CIPN is higher in patients whose dominant side is the breast cancer-affected side. It was suggested that excessive use of the breast cancer-affected side hand (dominant hand) in daily life may be a predictor for the development of CIPN. Further verification of this is needed.

Surgical glove size (small) and average difference (large) in fingertip temperature before and after administration were also predictors on univariate analysis. In other words, it was suggested that CIPN 
could be prevented if the glove pressure is strong, or the cooling temperature is low. This is consistent with the results of previous studies [6-8].

There were several limitations to the current study. First, both hands of the same patient were considered to be independent. Second, the methods of evaluating CIPN is subjective which may underestimate the real number of patients with CIPN. Third, since this study was conducted at a single institute, it only analyzed a relatively small number of patients. Therefore, a prospective, multicenter study will be needed to confirm these results.

In conclusion, smoking history, $\mathrm{BMI}$, and advanced age were identified as significant predictors of the development of CIPN in cancer patients treated with nab-PTX. CIPN may develop more easily in dominant hand on the breast cancer-affected side. However, these preliminary findings will need to be confirmed in a larger randomized, controlled trial. Nevertheless, these findings may assist in developing chemotherapeutic strategies, including of taxane chemotherapy, with better safety and efficacy, and to improve the quality of life of patients.

\section{Declarations}

Conflict of interest: None declared.

\section{Acknowledgements}

The authors would like to thank all the patients and medical staff at University Hospital, Kyoto Prefectural University of Medicine who were involved in this study.

\section{Author contributions}

Y.K. concept and design, data acquisition, data analysis, data interpretation, manuscript writing; K.S. and T.I. concept and design, data acquisition, data interpretation; Y.T. data acquisition, data interpretation; R.T.and I.Y, concept and design, data analysis; N.K. and K.T. concept and design; T.T. concept and design, data acquisition, data interpretation, supervision of the manuscript. All authors read and approved the final manuscript.

\section{Funding}

None.

\section{Data availability}

Data not available due to ethical restrictions.

Participants of this study did not agree for their data to be shared publicly, so supporting data is not available. 
Code availability N/A.

\section{Conflict of interest statement}

Isao Yokota has received a speaker's fee from Chugai Pharmaceutical Co., Ltd. Norito Katoh has been a speaker and investigator for Taiho Pharmaceutical Co., Ltd. Tetsuya Taguchi received a speaker's fee and research funds from Taiho Pharmaceutical and

Chugai Pharmaceutical Co., Ltd. All other authors declare that they have no conflicts of interest concerning this work.

\section{Ethics approval}

This trial was approved by the Medical Ethics Review Committee of Kyoto Prefectural University of Medicine, in accordance with the tenets of the Declaration of Helsinki (approval no. ERB-C-906-1).

\section{Informed consent}

Written, informed consent was obtained from all patients prior to their enrolment.

\section{Consent for publication}

Written, consent for publication was obtained from all patients prior to their enrolment.

\section{References}

1. Staff NP, Grisold A, Grisold W, Windebank AJ. Chemotherapy-induced peripheral neuropathy: A current review. Ann Neurol. 2017;81:772-81. doi:10.1002/ana.24951.

2. Wolf S, Barton D, Kottschade L, Grothey A, Loprinzi C. Chemotherapy-induced peripheral neuropathy: prevention and treatment strategies. Eur J Cancer. 2008;44:1507-15. doi:10.1016/j.ejca.2008.04.018.

3. Ewertz M, Qvortrup C, Eckhoff L. Chemotherapy-induced peripheral neuropathy in patients treated with taxanes and platinum derivatives. Acta Oncol. 2015;54:587-91. doi:10.3109/0284186X.2014.995775.

4. Mols F, Beijers T, Vreugdenhil G, van de Poll-Franse L. Chemotherapy-induced peripheral neuropathy and its association with quality of life: a systematic review. Support Care Cancer. 2014;22:2261-9. doi:10.1007/s00520-014-2255-7.

5. Speck RM, Sammel MD, Farrar JT, Hennessy S, Mao JJ, Stineman MG, DeMichele A. Impact of chemotherapy-induced peripheral neuropathy on treatment delivery in nonmetastatic breast cancer. J Oncol Pract. 2013;9:e234-40. doi:10.1200/JOP.2012.000863.

6. Hanai A, Ishiguro H, Sozu T, Tsuda M, Yano I, Nakagawa T, Imai S, Hamabe Y, Toi M, Arai H, Tsuboyama T. Effects of Cryotherapy on Objective and Subjective Symptoms of Paclitaxel-Induced 
Neuropathy: Prospective Self-Controlled Trial. J Natl Cancer Inst. 2018;110:141-8. doi:10.1093/jnci/djx178.

7. Tsuyuki S, Senda N, Kanng Y, Yamaguchi A, Yoshibayashi H, Kikawa Y, Katakami N, Kato H, Hashimoto T, Okuno T, Yamauchi A, Inamoto T. Evaluation of the effect of compression therapy using surgical gloves on nanoparticle albumin-bound paclitaxel-induced peripheral neuropathy: a phase II multicenter study by the Kamigata Breast Cancer Study Group. Breast Cancer Res Treat. 2016;160:61-7. doi:10.1007/s10549-016-3977-7.

8. Tsuyuki S, Yamagami K, Yoshibayashi H, Sugie T, Mizuno Y, Tanaka S, Kato H, Okuno T, Ogura N, Yamashiro H, Takuwa H, Kikawa Y, Hashimoto T, Kato T, Takahara S, Katayama T, Yamauchi A, Inamoto T. Effectiveness and safety of surgical glove compression therapy as a prophylactic method against nanoparticle albumin-bound-paclitaxel-induced peripheral neuropathy. Breast. 2019;47:22-7. doi:10.1016/j.breast.2019.06.008.

9. Kanbayashi Y, Sakaguchi K, Ishikawa T, Ouchi Y, Nakatsukasa K, Tabuchi Y, Kanehisa F, Hiramatsu M, Takagi R, Yokota I, Katoh N, Taguchi T. Comparison of the efficacy of cryotherapy and compression therapy for preventing nanoparticle albumin-bound paclitaxel-induced peripheral neuropathy: A prospective self-controlled trial. Breast. 2020;49:219-24. doi:10.1016/j.breast.2019.12.011.

10. Hiramoto S, Asano H, Miyamoto T, Takegami M, Kawabata A. Predictors and pharmacotherapy for chemotherapy-induced peripheral neuropathy in paclitaxel-treated female cancer survivors: A retrospective study in Japan. PLoS ONE. 2021;16:e0261473. doi:10.1371/journal.pone.0261473.

11. Ghoreishi Z, Keshavarz S, Asghari Jafarabadi M, Fathifar Z, Goodman KA, Esfahani A. Predictors for paclitaxel-induced peripheral neuropathy in patients with breast cancer. BMC Cancer. 2018;18:958. doi:10.1186/s12885-018-4869-5.

12. Miyamoto T, Hiramoto S, Kanto A, Tsubota M, Fujitani M, Fukuyama H, Hatanaka S, Sekiguchi F, Koizumi Y, Kawabata A. Estrogen decline is a predictor for paclitaxel-induced peripheral neuropathy: Clinical evidence supported by a preclinical study. J Pharmacol Sci. 2021;146:49-57. doi:10.1016/j.jphs.2021.03.001.

13. Loprinzi CL, Lacchetti C, Bleeker J, Cavaletti G, Chauhan C, Hertz DL, Kelley MR, Lavino A, Lustberg MB, Paice JA, Schneider BP, Lavoie Smith EM, Smith ML, Smith TJ, Wagner-Johnston N, Hershman DL. Prevention and Management of Chemotherapy-Induced Peripheral Neuropathy in Survivors of Adult Cancers: ASCO Guideline Update. J Clin Oncol. 2020;38:3325-48. doi:10.1200/JC0.20.01399.

14. Jordan B, Margulies A, Cardoso F, Cavaletti G, Haugnes HS, Jahn P, Le Rhun E, Preusser M, Scotté F, Taphoorn MJB, Jordan K, ESMO Guidelines Committee. Electronic address:

clinicalguidelines@esmo.org; EONS Education Working Group. Electronic address: eons.secretariat@cancernurse.eu; EANO Guideline Committee. Electronic address: office@eano.eu. Systemic anticancer therapy-induced peripheral and central neurotoxicity: ESMO-EONS-EANO Clinical Practice Guidelines for diagnosis, prevention, treatment and follow-up. Ann Oncol. 2020;31:1306-19. doi:10.1016/j.annonc.2020.07.003. 
15. Smith EM, Pang H, Cirrincione C, Fleishman S, Paskett ED, Ahles T, Bressler LR, Fadul CE, Knox C, LeLindqwister N, Gilman PB, Shapiro CL. Alliance for Clinical Trials in Oncology. Effect of duloxetine on pain, function, and quality of life among patients with chemotherapy-induced painful peripheral neuropathy: a randomized clinical trial. JAMA. 2013;309:1359-67. doi:10.1001/jama.2013.2813.

16. Shimozuma K, Ohashi Y, Takeuchi A, Aranishi T, Morita S, Kuroi K, Ohsumi S, Makino H, Mukai H, Katsumata N, Sunada Y, Watanabe T, Hausheer FH. Feasibility and validity of the Patient Neurotoxicity Questionnaire during taxane chemotherapy in a phase III randomized trial in patients with breast cancer: N-SAS BC 02. Support Care Cancer. 2009;17:1483-91. doi:10.1007/s00520-0090613-7.

17. Seretny M, Currie GL, Sena ES, Ramnarine S, Grant R, MacLeod MR, Colvin LA, Fallon M. Incidence, prevalence, and predictors of chemotherapy-induced peripheral neuropathy: A systematic review and meta-analysis. Pain. 2014;155:2461-70. doi:10.1016/j.pain.2014.09.020.

18. Lauritsen J, Bandak M, Kreiberg M, Skøtt JW, Wagner T, Rosenvilde JJ, Dysager L, Agerbæk M, Daugaard G. Long-term neurotoxicity and quality of life in testicular cancer survivors-a nationwide cohort study. J Cancer Surviv. 2021;15:509-17. doi:10.1007/s11764-020-00944-1.

19. Molassiotis A, Cheng HL, Leung KT, Li YC, Wong KH, Au JSK, Sundar R, Chan A, Ng TR, Suen LKP, Chan CW, Yorke J, Lopez V. Risk factors for chemotherapy-induced peripheral neuropathy in patients receiving taxane- and platinum-based chemotherapy. Brain Behav. 2019;9:e01312. doi:10.1002/brb3.1312.

20. Dolan ME, El Charif O, Wheeler HE, Gamazon ER, Ardeshir-Rouhani-Fard S, Monahan P, Feldman DR, Hamilton RJ, Vaughn DJ, Beard CJ, Fung C, Kim J, Fossa SD, Hertz DL, Mushiroda T, Kubo M, Einhorn LH, Cox NJ, Travis LB. Platinum Study Group. Clinical and Genome-Wide Analysis of CisplatinInduced Peripheral Neuropathy in Survivors of Adult-Onset Cancer. Clin Cancer Res. 2017;23:575768. doi:10.1158/1078-0432.CCR-16-3224.

21. Greenlee H, Hershman DL, Shi Z, Kwan ML, Ergas IJ, Roh JM, Kushi LH. BMI, Lifestyle Factors and Taxane-Induced Neuropathy in Breast Cancer Patients: The Pathways Study. J Natl Cancer Inst. 2016;109:djw206. doi:10.1093/jnci/djw206.

22. Timmins HC, Li T, Goldstein D, Trinh T, Mizrahi D, Harrison M, Horvath LG, Friedlander M, Kiernan MC, Park SB. The impact of obesity on neuropathy outcomes for paclitaxel- and oxaliplatin-treated cancer survivors. J Cancer Surviv. 2021. doi:10.1007/s11764-021-01012-y. (in press).

23. Petrovchich I, Kober KM, Wagner L, Paul SM, Abrams G, Chesney MA, Topp K, Smoot B, Schumacher M, Conley YP, Hammer M, Levine JD, Miaskowski C. Deleterious Effects of Higher Body Mass Index on Subjective and Objective Measures of Chemotherapy-Induced Peripheral Neuropathy in Cancer Survivors. J Pain Symptom Manage. 2019;58:252-63. doi:10.1016/j.jpainsymman.2019.04.029.

24. Bao T, Basal C, Seluzicki C, Li SQ, Seidman AD, Mao JJ. Long-term chemotherapy-induced peripheral neuropathy among breast cancer survivors: prevalence, risk factors, and fall risk. Breast Cancer Res Treat. 2016;159:327-33. doi:10.1007/s10549-016-3939-0. 
25. Mizrahi D, Park SB, Li T, Timmins HC, Trinh T, Au K, Battaglini E, Wyld D, Henderson RD, Grimison P, Ke H, Geelan-Small P, Marker J, Wall B, Goldstein D. Hemoglobin, Body Mass Index, and Age as Risk Factors for Paclitaxel- and Oxaliplatin-Induced Peripheral Neuropathy. JAMA Netw Open. 2021;4:e2036695. doi:10.1001/jamanetworkopen.2020.36695. 